\title{
Phenotypic plasticity of body pigmentation in Drosophila melanogaster. Genetic repeatability of quantitative parameters in two successive generations
}

\author{
P Gibert, B Moreteau and JR David \\ CNRS, Lab. Populations, Génétique, Evolution, Avenue de la Terrasse, 91198 Gif sur Yvette Cedex, France
}

\begin{abstract}
In Drosophila melanogaster, body pigmentation is a quantitative trait that depends on developmental temperature. When investigated over the whole thermal range of the species, pigmentation exhibits nonlinear reaction norms that differ among segments. The isofemale line method was used to analyse the genetic variability in two natural populations that affected the shape of reaction norms. Each line was considered as an experimental repeat, and polynomial reaction norms fitted to calculate the characteristic values (eg the coordinates of a maximum). In total, 20 lines from two geographically distant populations (France and India) were investigated at seven developmental temperatures (12$31^{\circ} \mathrm{C}$ ) in two successive generations (G2 and G3). We analysed the genetic repeatability (ie the correlation between generations) of three kinds of parameters: intraclass correla-
\end{abstract}

tion coefficients (isofemale heritability), family means at different temperatures and the characteristic values of the reaction norms. For intraclass correlation, a low genetic repeatability was found. For family mean values grown at various temperatures, an overall positive and highly significant repeatability was found $(r=0.55 \pm 0.024)$. Finally, a positive significant G2-G3 correlation was also the rule for the characteristic values of the reaction norms. Significant differences could be found between values describing either the trait or its plasticity, but with no general trend. A slightly higher repeatability was observed in the Indian population. These results show that, with a family selection design, the shape of the reaction norms might be modified in various ways. Heredity (2004) 92, 499-507, advance online publication, 24 March 2004; doi:10.1038/sj.hdy.6800449

Keywords: reaction norms; abdomen pigmentation; thorax pigmentation; isofemale line heritability; genetic correlation

\section{Introduction}

Phenotypic plasticity, the capacity of a single genotype to produce different phenotypes in different environments, receives increasing attention among evolutionary biologists (Via, 1992, 1993, 1994; Scheiner, 1993a, b; Schlichting and Pigliucci, 1993, 1998; Van Tienderen and Koelewijn, 1994; De Jong, 1995; Gotthard and Nylin, 1995; Via et al, 1995; Pigliucci, 1998, 2001; De Jong and Gavrilets, 2000; De Witt and Scheiner, 2003). Plasticity may be considered as a specific trait, and the evolution of plasticity is now a hotly debated subject among evolutionary biologists (Via et al, 1995; Callahan et al, 1997; Agrawal, 2001; van Dooren, 2001; Scheiner, 2002; David et al, 2003).

Previous investigations on size-related traits (Scheiner and Lyman, 1989) showed that plasticity was a specific property, independent of trait value, and could be increased or decreased by directional selection. These experiments used only two environments (two developmental temperatures) so that linear reaction norms were considered. However, when analysed over a broad

Correspondence: P Gibert, Current address: Lab. Biométrie, Biologie Evolutive, Bat. Gregor Mendel, Université Lyon 1-43, Bld du 11 novembre 1918, 69622 Villeurbanne Cedex, France.

E-mail: gibert@biomserv.univ-lyon1.fr

Received 29 November 2002; revised 10 July 2003; accepted 24 September 2003; published online 24 March 2004 thermal range, reaction norms of a range of quantitative traits, including size traits (David et al, 1994; Morin et al, 1996), ovariole number (Delpuech et al, 1995), bristle number (Moreteau et al, 2002) and body pigmentation (David et al, 1990; Gibert et al, 1996, 2000) are never linear. A general method for analysing and describing the shape of a reaction norm is a polynomial relationship and both quadratic or cubic forms can be used (David et al, 1997; Gibert et al, 1998a). Quadratic norms exhibit a maximum or minimum within the thermal range, while cubic norms generally exhibit a monotonically decreasing or increasing sigmoid shape.

Polynomial coefficients can be used to calculate, for a given kind of norm, characteristic values, which generally have a clear biological meaning. For example, a quadratic concave norm, which is mathematically defined by three polynomial coefficients, will be biologically described by three characteristic values, that is, the coordinates of its maximum and a curvature parameter. Interestingly, one value characterises the trait (maximum value) while the two others (curvature and temperature of maximum value) define its plasticity.

A characterisation of the phenotypic plasticity found in a natural population requires repeated measurement, and the method of isofemale lines (fullsib families) is a convenient and widespread design (Hoffmann and Parsons, 1988; Falconer, 1989). Significant differences between family means are generally considered to have a 
genetical basis, and intraclass correlation provides an estimate of the genetic variability (Capy et al, 1994). By extension, variations of characteristic values are assumed also to have a genetic basis (David et al, 1994; Morin et al, 1996).

Confidence in the existence of a genetic effect can, however, be gained only if a line keeps its phenotypic properties over successive generations (Krebs and Loeschke, 1995). This methodology was previously applied to wing length and female abdomen pigmentation in 37 lines over nine laboratory generations, grown at $25^{\circ} \mathrm{C}$. Results revealed a good correlation (called genetic repeatability) among family means, even between distant generations (Gibert et al, 1998b).

This procedure has been applied here in a similar way to the analysis of reaction norm shape of body pigmentation in female. This trait was chosen because it can be easily scored on a large number of flies. Different segments (thorax and abdomen) are known to exhibit quite different reaction norms (Gibert et al, 2000) and it was interesting to see if similar conclusions would be obtained for quadratic or cubic norms. Since $D$. melanogaster is known to exhibit a large amount of adaptive geographic differentiation (David and Capy, 1988; Munjal et al, 1997), we investigated two samples of 20 lines from two distant localities over two successive laboratory generations. We find that the characteristic values of the reaction norms are genetically repeatable and that there is no systematic difference between trait and plasticity characters. It should thus be possible to use these characteristic values for family directional selection and manipulate the shape of the reaction norm in different ways.

\section{Material and methods}

\section{Populations and experimental procedure}

Wild $D$. melanogaster females were collected with banana traps in Rohtak (India, about $70 \mathrm{~km}$ West of Delhi) and in Bordeaux (Southern France) and isolated in culture vials. These two localities were chosen for their contrasting climates. Bordeaux is characterised by a mild summer and a cold winter (see Gibert et al, 1996). Rohtak, on the other hand, is a subtropical place with a mild winter but a very hot summer (see Gibert et al, 1998c). We used these females to establish 20 isofemale lines for both populations. Lines were grown in culture vials at about $20^{\circ} \mathrm{C}$ under LD 16:8 h. After adult emergence, 10 pairs of the first laboratory generation were used as parents for each line. They oviposited at room temperature (about 20$22^{\circ} \mathrm{C}$ ) for half a day in vials containing a killed yeast medium, which reduces crowding effects (see Karan et al, 1999). Vials with eggs were then transferred at one of seven constant experimental temperatures $(12,14,17,21$, 25, 28 and $31^{\circ} \mathrm{C}$ ). On emergence, adults (second laboratory generation, G2) were transferred to fresh food and examined a few days later. From each line at each temperature, 10 females were randomly taken and measured.

We repeated the measurements on the next generation in order to analyse the genetic repeatability of phenotypic plasticity (see Gibert et al, 1998b). From the G2 flies grown at $21^{\circ} \mathrm{C}$, we randomly took 10 pairs as parents for the G3 and used the same experimental procedure, transferring culture vials at seven temperatures.

\section{Pigmentation scores}

We analysed pigmentation on the mesonotum (thoracic trident) and on all abdominal segments (segments 2-7) in females. Pigmentation was estimated using phenotypic classes ranging from 0 (completely yellow) to 10 (completely dark) for abdominal segment, and from 0 (no visible trident) to 3 (dark trident) for thoracic trident (see David et al, 1985, 1990 for details). For comparing thorax and abdominal pigmentation, we standardised their possible range of variation. The trident pigmentation score was thus multiplied by 3.33 , so that its variability ranged also between 0 and 10 .

\section{Data analysis}

Reaction norm shape was analysed by fitting a polynomial relationship (David et al, 1997; Gibert et al, 1998a). Depending on the shape, either a quadratic or cubic polynomial was used and coefficients were calculated with SAS software (SAS, 1985). The polynomial coefficients were then used to calculate characteristic values of the norms providing information either on traits (pigmentation) or on plasticity (see David et al, 1997; Gibert et al, 2000). All other statistics were calculated with Statistica software (Statistica, 1999). A general analysis was carried out by a four-way ANOVA with population, generation and temperature considered as fixed effects and lines as random effect nested within population.

\section{Results}

\section{Average reaction norms}

Average reaction norms of six abdomen segments (2-7) are shown in Figure 1. The figure illustrates the fact that different segments exhibit different reaction norms that differ in their average value, their shape and the magnitude of variation (Gibert et al, 2000). More precisely, segments 2, 3, 4 and 5 exhibit quadratic, convex norms, while segments 6 and 7 follow decreasing sigmoid curves. With respect to growth temperature, segment 2 is the least variable, segment 7 the most variable. Reaction norms of the thoracic trident (not shown) also exhibit a convex shape with a broader amplitude.

These curves were analysed by ANOVA (not shown). For the sake of simplicity, we present only the percentages of variation explained for each effect (Table 1). A main conclusion is that the generation effect was not significant for any segment. In each case there were significant differences among lines, which evidence a genetic variability within population. The population effects were all significant and explained, on average, $3.8 \%$ of the variation. This corresponds to a lighter pigmentation in the Indian population (see Gibert et al, 1998c). Finally, the largest effect was due to temperature (on average 61\%). Interestingly, phenotypic plasticity due to temperature was variable across segments and increased toward the posterior end of the abdomen (see also Gibert et al, 2000). 

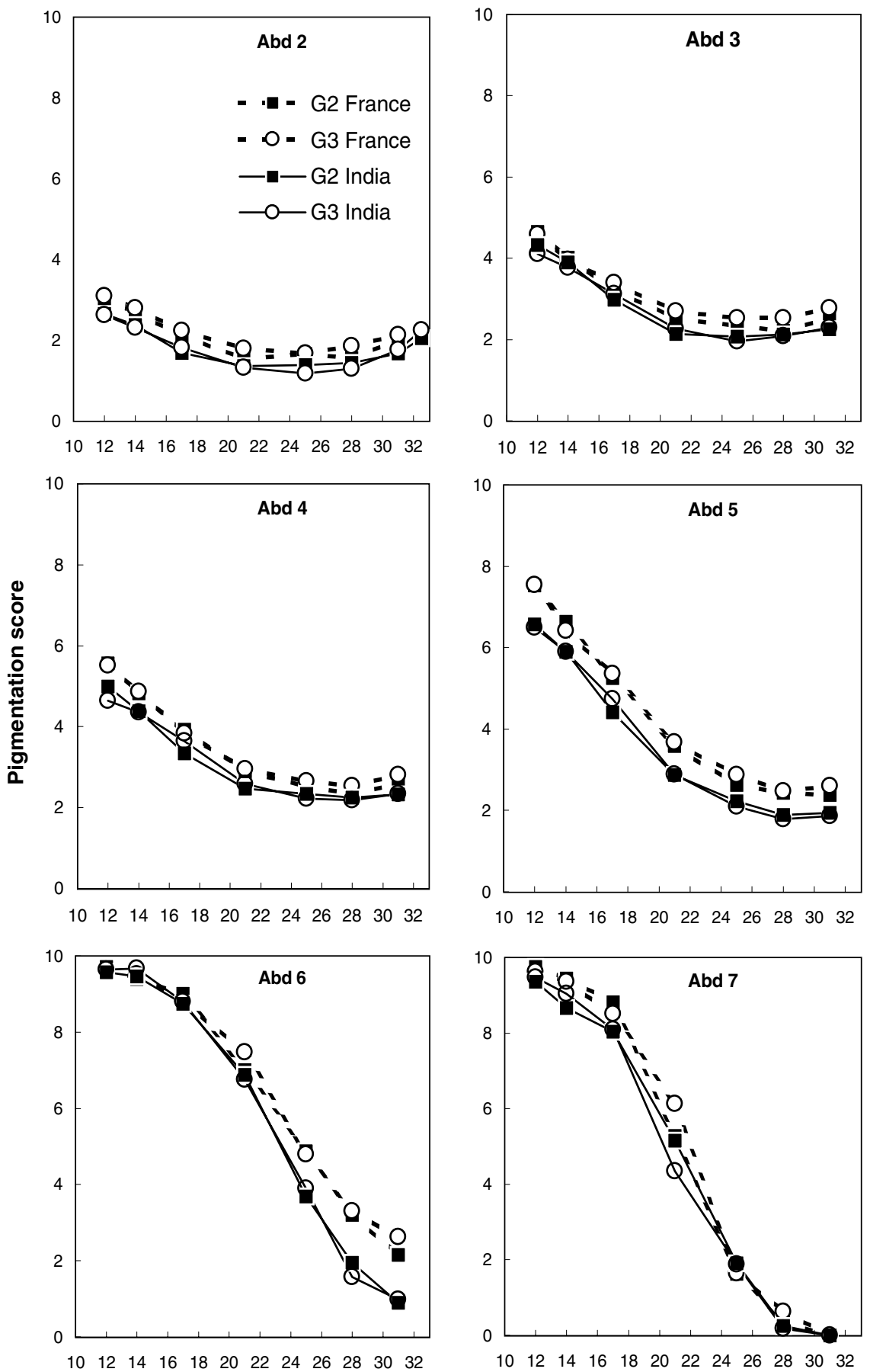

Developmental temperature $\left({ }^{\circ} \mathrm{C}\right)$

Figure 1 Reaction norms of abdomen segments 2-7 according to developmental temperature for the two successive generations (G2 and G3) and the two populations (France and India).

Genetic variability in the two successive generations The amount of genetic divergence between lines was estimated for each segment, temperature, population and generation by the coefficient of intraclass correlation $t$ (Hoffmann and Parsons, 1988). Overall, 196 coefficients were calculated: mean values are given in Table 2 and results of an ANOVA are presented in Table 3. Significant differences were found for two main effects and four double interactions.
The major effect was due to differences between segments $(19.2 \%$ of total variation). The genetic variability was less for the four anterior segments (thorax and Abd. $2,3,4)$ being on average $0.24 \pm 0.02$, and higher for Abd 5, 6, 7 (on average 0.32 \pm 0.02 ).

We also found significant differences between generations $(P=0.0013)$ : intraclass correlations were higher in G3 than in G2 $(0.294 \pm 0.021$ vs $0.256 \pm 0.015)$. No significant differences were found however between 
Table 1 Percentage of pigmentation variation explained by the main effects after ANOVA

\begin{tabular}{lccccccc}
\hline & Thorax $(\%)$ & $A b d 2(\%)$ & $A b d 3(\%)$ & $A b d 4(\%)$ & $A b d 5(\%)$ & $A b d 6(\%)$ & $A b d 7(\%)$ \\
\hline Population & $14.75^{* * *}$ & $4.31^{* * *}$ & $1.86^{* *}$ & $2.13^{* * *}$ & $2.60^{* * *}$ & $0.90^{* * *}$ \\
Generation & $0.00 \mathrm{NS}$ & $0.08 \mathrm{NS}$ & $0.06 \mathrm{NS}$ & $0.01 \mathrm{NS}$ & $0.00 \mathrm{NS}$ & $0.01 \mathrm{NS}$ & $0.17^{* *}$ \\
Temperature & $40.81^{* * *}$ & $30.42^{* * *}$ & $48.82^{* * *}$ & $58.34^{* * *}$ & $73.83^{* * *}$ & $85.08^{* * *}$ \\
Lines & $5.08^{* * *}$ & $8.53^{* * *}$ & $6.21^{* * *}$ & $4.04^{* * *}$ & $3.44^{* * *}$ & $2.15^{* * *}$ & $90.50^{* * *}$ \\
Interactions & 8.68 & 14.94 & 9.81 & 8.12 & 5.50 & 4.03 & $2.77^{* * *}$ \\
Error & 30.69 & 41.72 & 33.23 & 27.37 & 14.63 & 7.83 \\
\hline
\end{tabular}

***P<0.001, ${ }^{* * P}<0.01, * P<0.05$, NS: nonsignificant.

Population, generation and temperature are considered as fixed effects and lines as random effect nested in population. Significance level (F value) is also given in each case. The effects of all interactions are (not significant) pooled.

Table 2 Mean values $( \pm S E$ ) of intraclass correlation averaged over the seven developmental temperatures for each segment, population and generation

\begin{tabular}{|c|c|c|c|c|c|c|c|}
\hline \multirow[t]{2}{*}{ Trait } & \multicolumn{2}{|c|}{ France } & \multicolumn{2}{|c|}{ India } & \multirow[b]{2}{*}{$M e a n \pm S E$} & \multicolumn{2}{|c|}{ Genetic repeatability } \\
\hline & G2 & G3 & G2 & G3 & & France & India \\
\hline Thorax & $0.23 \pm 0.04$ & $0.24 \pm 0.03$ & $0.15 \pm 0.05$ & $0.13 \pm 0.05$ & $0.19 \pm 0.02$ & 0.28 & -0.25 \\
\hline Abd 2 & $0.31 \pm 0.05$ & $0.30 \pm 0.05$ & $0.23 \pm 0.03$ & $0.27 \pm 0.04$ & $0.28 \pm 0.02$ & 0.77 & -0.03 \\
\hline Abd 3 & $0.19 \pm 0.02$ & $0.27 \pm 0.06$ & $0.31 \pm 0.04$ & $0.29 \pm 0.04$ & $0.26 \pm 0.02$ & 0.37 & -0.55 \\
\hline Abd 4 & $0.19 \pm 0.02$ & $0.29 \pm 0.05$ & $0.23 \pm 0.04$ & $0.26 \pm 0.02$ & $0.24 \pm 0.02$ & 0.38 & -0.90 \\
\hline Abd 5 & $0.24 \pm 0.03$ & $0.38 \pm 0.02$ & $0.27 \pm 0.02$ & $0.31 \pm 0.04$ & $0.30 \pm 0.03$ & -0.05 & 0.35 \\
\hline Abd 6 & $0.30 \pm 0.05$ & $0.39 \pm 0.03$ & $0.35 \pm 0.05$ & $0.43 \pm 0.03$ & $0.37 \pm 0.02$ & 0.18 & -0.25 \\
\hline Abd 7 & $0.32 \pm 0.05$ & $0.34 \pm 0.03$ & $0.26 \pm 0.05$ & $0.24 \pm 0.04$ & $0.29 \pm 0.02$ & -0.46 & 0.89 \\
\hline Mean \pm SE & $0.25 \pm 0.02$ & $0.32 \pm 0.02$ & $0.25 \pm 0.02$ & $0.28 \pm 0.03$ & & $0.21 \pm 0.14$ & $-0.11 \pm 0.22$ \\
\hline
\end{tabular}

Abd 2 to 7: abdominal pigmentation for tergites 2 to 7; thorax: thoracic pigmentation.

Genetic repeatability is calculated as the correlation between values of G2 and G3. Each coefficient is based on seven observations. The significance threshold is 0.75 for $P=0.05$.

Table 3 ANOVA on intraclass correlation coefficients

\begin{tabular}{lrccc}
\hline Source & $d f$ & $M S$ & $F$ value & \% explained variance \\
\hline Segment (1) & 6 & 0.084 & $12.12^{* * *}$ & 19.2 \\
Population (2) & 1 & 0.014 & $2.03 \mathrm{NS}$ & 2.9 \\
Generation (3) & 1 & 0.078 & $11.24^{* *}$ & 0.5 \\
Temperature (4) & 6 & 0.007 & $1.00 \mathrm{NS}$ & 1.6 \\
$1^{* 2}$ & 6 & 0.022 & $3.22^{* *}$ & 5.1 \\
$1^{* 3}$ & 6 & 0.009 & $1.43 \mathrm{NS}$ & 2.2 \\
$1^{* 4}$ & 35 & 0.013 & $1.90^{*}$ & 17.6 \\
$2^{*} 3$ & 1 & 0.024 & $3.51 \mathrm{NS}$ & 0.9 \\
$2^{* 4}$ & 6 & 0.020 & $2.88^{*}$ & 4.6 \\
$3^{* 4}$ & 6 & 0.042 & $6.01^{* * *}$ & 9.5 \\
$1^{*} 2^{* 3}$ & 6 & 0.005 & $0.71 \mathrm{NS}$ & 1.1 \\
$1^{*} 2^{* 4}$ & 35 & 0.010 & $1.46 \mathrm{NS}$ & 13.5 \\
$2^{*} 3^{* 4}$ & 6 & 0.012 & $1.79 \mathrm{NS}$ & 2.8 \\
Error & 69 & 0.0069 & & \\
\hline
\end{tabular}

***P<0.001, **P<0.01, NS: nonsignificant.

All factors are considered as fixed. df: degree of freedom; MS: mean square; F: variance ratio.

populations $(P=0.16)$ or temperatures $(P=0.43)$. The absence of a direct temperature effect justifies the calculation of mean values averaged over temperatures in Table 2. Most double interactions (four out of six) were, however, significant and the major effects concern population-temperature and generation-temperature interactions. These results suggest a complex genetic architecture of body pigmentation in D. melanogaster.

We also analysed the genetic repeatability of intraclass correlations $(t)$ : Pearson's correlation coefficients between G2 and G3 values were calculated across temperatures for each trait and population. Results (Table 2) proved to be extremely variable with a few significant values but, surprisingly, both positive (Abd 7, India, $r=0.89$ ) and negative (eg Abd 4, India, $r=-0.90$ ). This suggests that variation of intraclass correlations, when analysed in this way, are not repeatable. This might be related to the fact that variation due to the direct effect of temperature is not significant (Table 3), while temperature is significantly involved in three interactions.

\section{Genetic repeatability of family mean pigmentation}

The method for analyzing the genetic repeatability is to calculate the correlation between the mean values of the lines across successive generations (Gibert et al, 1998b). These correlations were calculated for each segment, temperature and population (Table 4), and a first general conclusion is that $71 \%$ of the correlations between the two successive generations were significantly positive (68 out of 96 coefficients). Moreover, only nine coefficients among 96 had a very low value, less than 0.1 .

We analysed the variability of correlations by ANOVA (not shown) after a $z$ transformation with three main fixed effects (population, temperature, segments). Owing to the absence of variance (pigmentation totally light with a score of zero), it was impossible to calculate a correlation in both populations for the abdominal segment 7 at $31^{\circ} \mathrm{C}$. Consequently, to get a balanced design, we removed all $31^{\circ} \mathrm{C}$ values from the ANOVA.

No significant differences were found between populations $(P=0.73)$ or temperature $(P=0.39)$ and no interaction was significant. The only significant difference was due to segments $(P=0.0075)$. Abd 5 and Abd 6 showed a higher genetic repeatability $(>0.6)$. For thorax and $\mathrm{Abd} 2$ to $\mathrm{Abd} 4$, the values were intermediate 
Table 4 Correlation coefficients between G2 and G3 (repeatability among family means) for each growth temperature and population ( $n=20$ in each case)

\begin{tabular}{|c|c|c|c|c|c|c|c|c|}
\hline Population & Temperature & Thorax & $A b d 2$ & $A b d 3$ & Abd 4 & Abd 5 & Abd 6 & Abd 7 \\
\hline \multirow[t]{7}{*}{ France } & 12 & $0.64^{* *}$ & $0.75^{* * *}$ & $0.63^{* *}$ & $0.29 \mathrm{NS}$ & $0.29 \mathrm{NS}$ & $0.72^{* * *}$ & $0.73^{* * *}$ \\
\hline & 14 & $0.19 \mathrm{NS}$ & $0.74^{* * *}$ & $0.70^{* *}$ & $0.53^{*}$ & $0.60^{*}$ & $0.87^{* * *}$ & $0.83^{* * *}$ \\
\hline & 17 & $0.59^{*}$ & $0.61^{* *}$ & $0.61^{* *}$ & $0.60^{*}$ & $0.77^{* * *}$ & $0.90^{* * *}$ & $0.77^{* * *}$ \\
\hline & 21 & $0.51^{*}$ & $0.10 \mathrm{NS}$ & $0.45 \mathrm{NS}$ & $0.60 \mathrm{NS}$ & $0.68^{* *}$ & $0.63^{* *}$ & $0.31 \mathrm{NS}$ \\
\hline & 25 & $0.85^{* * *}$ & $0.49^{*}$ & $0.54^{*}$ & $0.57^{*}$ & $0.47 \mathrm{NS}$ & $0.60^{*}$ & $0.48^{*}$ \\
\hline & 28 & $0.48 \mathrm{NS}$ & $0.58^{*}$ & $0.53^{*}$ & $0.51^{*}$ & $0.71^{* * *}$ & $0.62^{* *}$ & $0.01 \mathrm{NS}$ \\
\hline & 31 & $0.42 \mathrm{NS}$ & $0.43 \mathrm{NS}$ & $0.09 \mathrm{NS}$ & $0.30 \mathrm{NS}$ & $0.46 \mathrm{NS}$ & $0.47 \mathrm{NS}$ & - \\
\hline \multirow[t]{9}{*}{ India } & 12 & $0.48^{*}$ & $0.03 \mathrm{NS}$ & $0.65^{* *}$ & $0.56^{*}$ & $0.67^{* *}$ & $0.79 * * *$ & $0.13 \mathrm{NS}$ \\
\hline & 14 & $0.83^{* * *}$ & $0.51^{*}$ & $0.58^{*}$ & $0.63^{* *}$ & $0.78^{* * *}$ & $0.87^{* * *}$ & $-0.29 \mathrm{NS}$ \\
\hline & 17 & $0.64^{* *}$ & $0.39 \mathrm{NS}$ & $0.05 \mathrm{NS}$ & $-0.03 \mathrm{NS}$ & $0.35 \mathrm{NS}$ & $0.80^{* * *}$ & $0.86^{* * *}$ \\
\hline & 21 & $0.82^{* * *}$ & $0.62^{* *}$ & $0.71^{* * *}$ & $0.71^{* * *}$ & $0.74^{* * *}$ & $0.90^{* * *}$ & $0.41 \mathrm{NS}$ \\
\hline & 25 & $0.58^{*}$ & $0.59^{*}$ & $0.56^{*}$ & $0.65^{* *}$ & $0.83^{* * *}$ & $0.74^{* * *}$ & $0.41 \mathrm{NS}$ \\
\hline & 28 & $0.50^{*}$ & $0.62^{* *}$ & $0.23 \mathrm{NS}$ & $0.41 \mathrm{NS}$ & $0.69^{* *}$ & $0.57^{*}$ & $0.05 \mathrm{NS}$ \\
\hline & 31 & $0.81^{* * *}$ & $0.55^{*}$ & $0.52^{*}$ & $0.61^{* *}$ & $0.58^{*}$ & $0.57^{*}$ & - \\
\hline & Mean & 0.596 & 0.501 & 0.489 & 0.496 & 0.616 & 0.718 & 0.392 \\
\hline & SE & 0.048 & 0.054 & 0.055 & 0.051 & 0.043 & 0.036 & 0.101 \\
\hline
\end{tabular}

We used a sequential Bonferroni-type procedure to correct critical $\mathrm{P}$-values (Benjamini and Hochberg, 1995 ). Correlations for Abd 7 at $31^{\circ} \mathrm{C}$ were not calculated due to a complete absence of dark pigmentation in most lines.
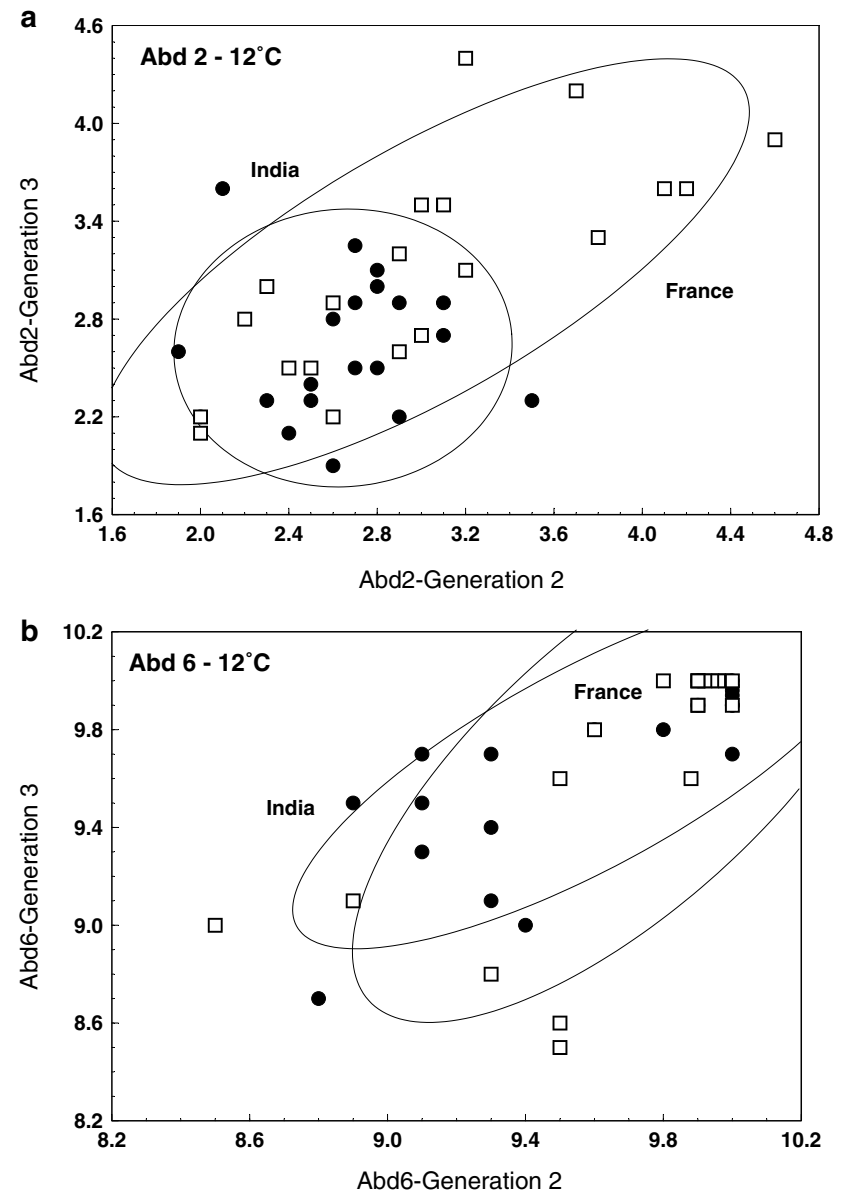

Figure 2 Relationship between the two successive generations (G2 and G3) for abdomen segments 3 (a) and 6 (b) at $12^{\circ} \mathrm{C}$. Each point corresponds to an isofemale line, that is, mean of 10 individuals. The $85 \%$ confidence interval ellipses are also given to help visualise the distributions.

(ranging from 0.489 to 0.596). Abd 7 exhibited a low repeatability (0.392 on average) and large variations across temperatures (see Table 4).
The general conclusion is that mean pigmentation scores of the same lines in two successive generations are positively correlated (overall mean $r=0.55 \pm 0.024$, $n=96$ ), or in other words, there is a highly significant genetic repeatability. Interestingly, the repeatability appears to be quite independent of the mean pigmentation score. We illustrate this genetic repeatability by two graphs (Figure 2) both corresponding to values obtained at $12{ }^{\circ} \mathrm{C}$. For segment 2 (low pigmentation) the high positive correlation in French lines contrasts with a complete absence of correlation in Indian lines. For Abd 6 , on the other hand, all lines are very dark and exhibit a strong positive correlation between the two successive generations.

\section{Genetic repeatability of reaction norms characteristic values}

Reaction norms of pigmentation according to developmental temperature were analysed by calculating the characteristic values of the appropriate polynomial relationship.

For thoracic trident and abdomen segments 2-5, which show U-shaped, convex reaction norms, a quadratic polynomial was used and three characteristic values were estimated for each line. The minimum pigmentation $(\mathrm{minP})$ characterises the trait investigated, in that case the pigmentation of each segment in each isofemale line. Curvature $\left(\mathrm{g}_{2}\right)$ and temperature of minimum pigmentation (TminP) characterise the reactivity of the trait to developmental temperature and thus describe its plasticity. In two cases out of 400 (line 2 for Abd 4 in Bordeaux G3 and line 1 for Abd 5 in India G3) it was impossible to obtain an appropriate fit and these lines were excluded from the calculations.

For each characteristic value, we calculated the correlation over the two generations and coefficients are given in Table 5. These coefficients were submitted to a three-way ANOVA (not shown) after a $z$ transformation.

No significant differences were found between segments. Slight but significant differences $(P=0.041)$ were 
Table 5 Correlation between characteristic values of G2 and G3 for segments that exhibit quadratic convex reaction norms

\begin{tabular}{|c|c|c|c|c|}
\hline Population & Trait & $M P$ & $\operatorname{TmP}$ & G2 \\
\hline \multirow[t]{5}{*}{ France } & Thorax $(n=20)$ & $0.767^{* * *}$ & 0.111 NS & -0.065 NS \\
\hline & Abd $2(n=20)$ & $0.547^{*}$ & $0.271 \mathrm{NS}$ & 0.447 NS \\
\hline & $\operatorname{Abd} 3(n=20)$ & $0.541^{*}$ & $-0.030 \mathrm{NS}$ & $0.314 \mathrm{NS}$ \\
\hline & $\operatorname{Abd} 4(n=19)$ & $0.381 \mathrm{NS}$ & -0.0075 NS & $0.382 \mathrm{NS}$ \\
\hline & Abd $5(n=20)$ & -0.174 NS & 0.147 NS & $0.681^{*}$ \\
\hline \multirow[t]{6}{*}{ India } & Thorax $(n=20)$ & $0.724^{* * *}$ & $0.233 \mathrm{NS}$ & $0.448 \mathrm{NS}$ \\
\hline & Abd $2(n=20)$ & $0.803^{* * *}$ & 0.176 NS & $0.386 \mathrm{NS}$ \\
\hline & $\operatorname{Abd} 3(n=20)$ & $0.629^{*}$ & $0.514^{*}$ & $0.636^{*}$ \\
\hline & $\operatorname{Abd} 4(n=20)$ & $0.549^{*}$ & $0.200 \mathrm{NS}$ & $0.533^{*}$ \\
\hline & Abd $5(n=19)$ & $0.672^{*}$ & $0.244 \mathrm{NS}$ & $0.623^{*}$ \\
\hline & Mean \pm SE & $0.544 \pm 0.085$ & $0.186 \pm 0.046$ & $0.438 \pm 0.064$ \\
\hline
\end{tabular}

MP: minimum pigmentation; TmP: temperature of minimum pigmentation; $\mathrm{g}_{2}$ : curvature.

We used a sequential Bonferroni-type procedure to correct critical $P$-values (Benjamini and Hochberg, 1995).

found between populations: correlations were higher in India than in France $(0.485 \pm 0.051$ vs $0.288 \pm 0.073)$. The main effect ( $37 \%$ of total variability) was due to differences between characteristic values. The trait value (minP) showed the highest genetic repeatability (on average $0.544 \pm 0.085)$. The curvature parameter, $\mathrm{g}_{2}$, also showed a significant repeatability $(0.438 \pm 0.064)$ while a very low repeatability $(0.186 \pm 0.046)$ was found for the other plasticity parameter, TminP.

These results are illustrated for thoracic trident in Figure 3. For the trait value (minP) a clear positive correlation was found in the two populations, although the mean values are very different: French lines are much darker than Indian ones. For the temperature of minimum pigmentation, on the other hand, correlations were very low: results of the G2 generation do not permit a prediction of the next generation.

For abdominal segments 6 and 7, which exhibit a decreasing sigmoid shape, a third degree polynomial was used and many more characteristic values can be calculated (David et al, 1997), such as the coordinates of the inflection point, the coordinates of a maximum and a minimum and the slope at the inflection point. Three of these values characterise the trait (minimum pigmentation, maximum pigmentation, pigmentation at the inflection point) and four its plasticity (temperatures at the minimum, maximum and inflection point, slope at the inflection point). For Abd 6, it was impossible to adjust the data to this model for three lines (line 8 in Bordeaux G2 and G3, lines 13 and 15 in Bordeaux G2).

Results in Table 6 show that among 28 calculated coefficients, 11 were significantly positive (after a Bonferroni correction), while six were very low (less than 0.1). These data were submitted to ANOVA (not shown) after a $z$ transformation. No significant difference was found between segments or characteristic values. A slight but significant population effect was found $(P=0.02)$ : repeatability was slightly better for the Indian population.

When averaged over populations and segments, correlations for different characteristic values ranged from 0.229 to 0.582 . A general trend is that higher (but nonsignificantly so) values were found for plasticity than for traits themselves (0.332 vs 0.473).

Segments 6 and 7 show similar sigmoid norms and moreover mean values are genetically correlated (Gibert et al, 2000). We added the results of segments 6 and 7 and

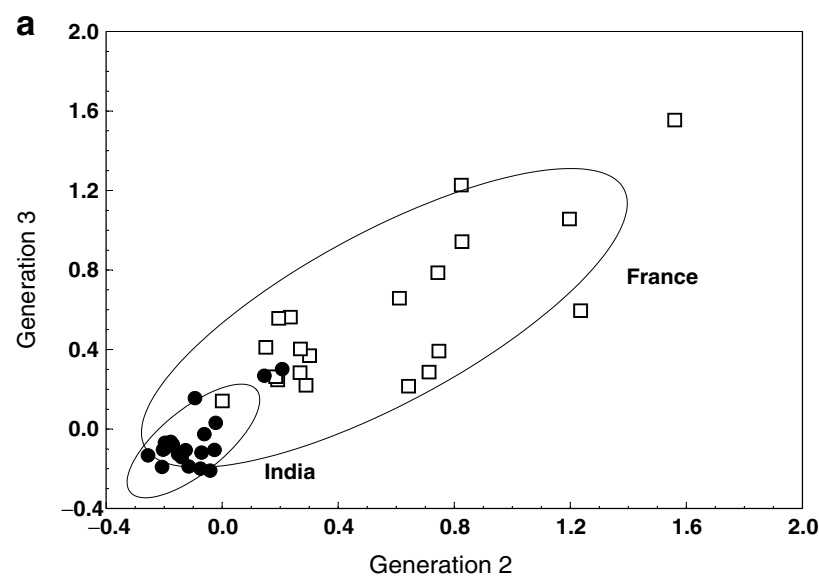

b

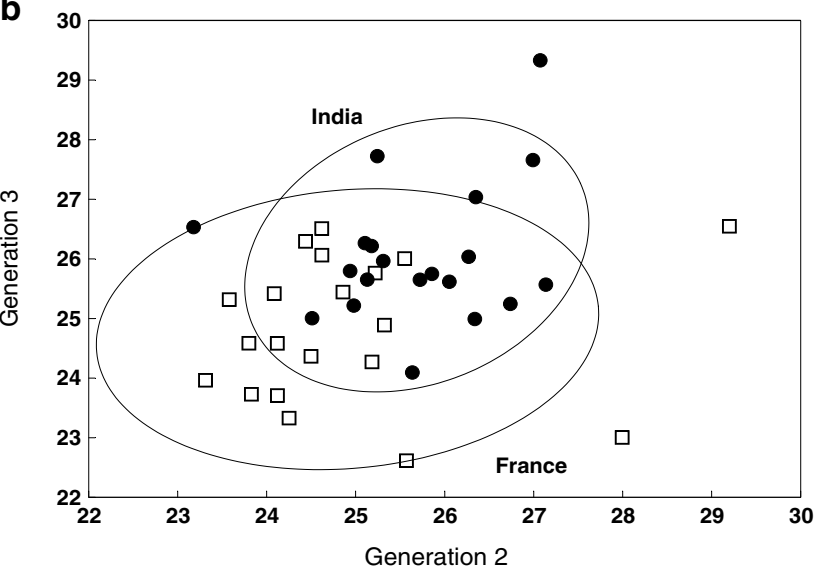

Figure 3 Relationship between the two successive generations (G2 and G3) for two characteristic values of the thoracic trident: the minimum pigmentation $(\operatorname{minP})(\mathbf{a})$ and the temperature of minimum pigmentation (TminP) (b). Each point corresponds to an isofemale line. The $85 \%$ confidence interval ellipses are also given.

considered the reaction norm of their sum, with the hope that a more regular conclusion could be obtained, and especially a better genetic repeatability. Results failed to confirm this expectation: correlations for the sum were generally intermediate between those of segments 6 and 7. We illustrate for the sum the genetic repeatability of two parameters (Figure 4): the maximum pigmentation, with correlations of 0.753 and 0.470 and the slope at 
Table 6 Correlation between successive generations for characteristic values calculated with a cubic polynomial

\begin{tabular}{|c|c|c|c|c|c|c|c|c|}
\hline \multirow[t]{2}{*}{ Population } & \multirow[t]{2}{*}{ Segment } & \multicolumn{3}{|c|}{ Trait characteristic values } & \multicolumn{4}{|c|}{ Plasticity characteristic values } \\
\hline & & $\min P$ & $\operatorname{MaxP}$ & Pip & $\operatorname{Tmin} P$ & $\operatorname{Tmax} P$ & Tip & Sip \\
\hline \multirow{2}{*}{ France } & Abd 6 & $0.603^{*}$ & $0.676^{* *}$ & 0.597 NS & $0.141 \mathrm{NS}$ & $0.275 \mathrm{NS}$ & $0.268 \mathrm{NS}$ & $0.596^{*}$ \\
\hline & Abd 7 & $-0.085 \mathrm{NS}$ & $-0.099 \mathrm{NS}$ & $0.073 \mathrm{NS}$ & $0.126 \mathrm{NS}$ & $0.518 \mathrm{NS}$ & $0.480 \mathrm{NS}$ & $0.473 \mathrm{NS}$ \\
\hline \multirow[t]{2}{*}{ India } & Abd 6 & 0.419 NS & $0.795^{* * *}$ & $0.777^{* * *}$ & $0.640^{* *}$ & $0.431 \mathrm{NS}$ & $0.674^{* *}$ & $0.775^{* * *}$ \\
\hline & Abd 7 & $-0.021 \mathrm{NS}$ & $0.782^{* * *}$ & $-0.067 \mathrm{NS}$ & $0.289 \mathrm{NS}$ & $0.744^{* * *}$ & $0.522 \mathrm{NS}$ & $0.484 \mathrm{NS}$ \\
\hline Mean \pm SE & & $0.229 \pm 0.145$ & $0.538 \pm 0.185$ & $0.360 \pm 0.167$ & $0.299 \pm 0.103$ & $0.492 \pm 0.085$ & $0.486 \pm 0.072$ & $0.582 \pm 0.061$ \\
\hline
\end{tabular}

minP: minimum pigmentation; maxP: maximum pigmentation; Pip: pigmentation at the inflexion point; TminP: temperature of minimum pigmentation; TmaxP: temperature of maximum pigmentation; Tip: temperature at the inflexion point; Sip: slope at the inflexion point. ${ }_{* * * P} P<0.001,{ }^{* *} P<0.01, * P<0.05$, NS: nonsignificant.

We used a sequential Bonferroni-type procedure to correct critical $P$-values (Benjamini and Hochberg, 1995).
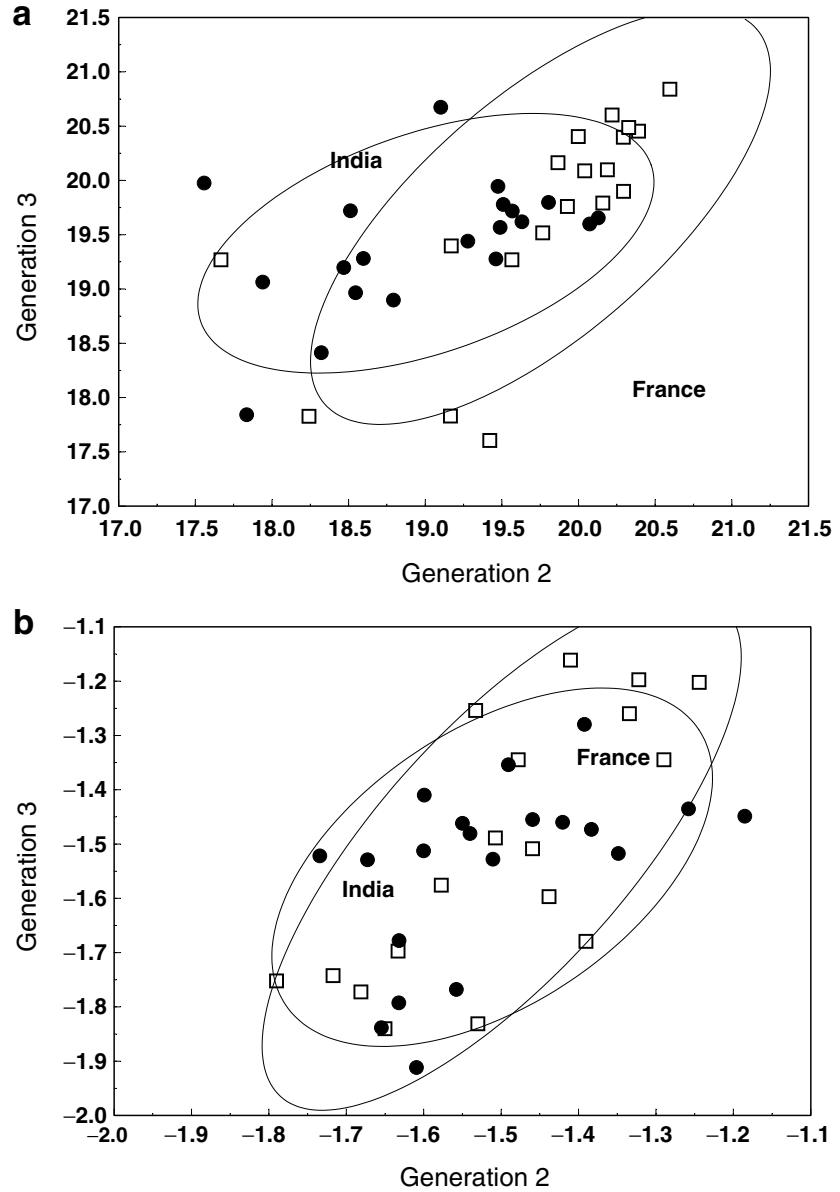

Figure 4 Relationship between the two successive generations (G2 and G3) for two characteristic values of the sum (Abd 6+Abd 7): the maximum pigmentation $(\operatorname{maxP})(a)$ and the slope at the inflection point (Sip) (b). Each point corresponds to an isofemale line. The $85 \%$ confidence interval ellipse is also given.

inflection point, with correlations of 0.753 and 0.498 in France and India respectively.

\section{Discussion}

Our data have confirmed previous observations, that for abdomen segments, there is an antero-posterior gradient of phenotypic plasticity correlated with an increasingly darker pigmentation (Gibert et al, 2000). There is also a significant difference between French and Indian populations, the latter being on average, lighter (Gibert et al, 1998c). This agrees also with previous data and is in accordance with the thermal budget hypothesis (Gibert et al, 1996): producing darker phenotypes in a cold environment should provide a functional advantage (favouring the absorption of light radiation) while the reverse is true in a much warmer climate, such as in India. Notice that the French-Indian difference is stronger for thorax pigmentation, a trait for which adaptive latitudinal clines are well documented in many geographical populations (David et al, 1985; Munjal et al, 1997).

Within each population and generation, calculation of an intraclass correlation $(t)$ demonstrates the occurrence of a genetic variability among lines. A first conclusion is that $t$ is variable among segments, increasing from the thorax toward the end of the abdomen. This phenomenon is difficult to interpret. It may reflect some real differences in genetic variability of successive segments, but it could also arise from an experimental bias. Anterior segments have a lower average pigmentation and measurement errors may be proportionately larger. Such a phenomenon was clearly demonstrated for wing dimensions (Imasheva et al, 2000). An artificial increase in the phenotypic within line variance could decrease intraclass correlation.

A second interesting observation is the overall significant increase of intraclass correlation in the second experimental generation, which is the third grown in the laboratory. There is no obvious explanation for this difference. A possibility could be that genetic drift has increased the variability among lines, producing a higher intraclass correlation. This interpretation is however unlikely since, in another experiment (Gibert et al, 1998b) where the pigmentation of 37 lines was measured in nine laboratory generations, there was only a very slight increase of the genetic variance over time.

There was no direct effect of growth temperature upon genetic variability. Temperature was however involved in three significant interactions (Table 3) with segment, population and generation, indicating that the genetic architecture of pigmentation may indeed be sensitive to thermal conditions. The relatively low influence of developmental temperature may explain the lack of an overall trend in genetic repeatability found in Table 3. 
Considering the mean pigmentation values in G2 and G3 (Table 4), we found an overall positive correlation since 68 out of 96 coefficients were significant (overall average $r=0.55 \pm 0.024)$. Surprisingly, a few correlation coefficients were very close to zero: however, the distribution of these coefficients did not show any regular pattern (Table 4). The only significant effect revealed by an appropriate ANOVA was a difference between segments. Genetic repeatability for the three anterior segments was less (on average $0.49 \pm 0.03$ ) than for thorax and segments 5 and 6 (average $0.64 \pm 0.03$ ). It might be argued, as for the intraclass correlation, that a lesser mean pigmentation increases the measurement error and decreases the repeatability. But this argument does not hold for abdomen 7 for which the repeatability was the lowest $(r=0.39)$. Basically, these data confirm a previous investigation in which 37 lines were measured over nine laboratory generations, but only for Abd 5, 6 and 7 , and at a single temperature $\left(25^{\circ} \mathrm{C}\right)$.

Genetic repeatability was also investigated for the shape of reaction norms, and to our knowledge this is the first time that such an investigation has been undertaken. In this case, two kinds of norms and also two kinds of shape descriptors (characteristic values) were considered. For five successive segments (thorax and abdomen 2-5) plasticity could be described by a quadratic norm and three values, one concerning the trait (minP) and two its plasticity (TminP and $\mathrm{g}_{2}$ ). For $\operatorname{minP}$, we found an overall positive correlation $(0.539 \pm 0.023)$ with a large variability between segments and populations (Table 5). This average repeatability is similar to that found for the mean values of lines $(r=0.55 \pm 0.024)$.

For the two plasticity parameters, correlations were still lower. For the TminP, the average correlation $(0.186 \pm 0.046)$ was very low. Clearly, knowing the temperature at which the lighter pigmentation is observed does not predict the value in the next generation. The situation was slightly better for the $g_{2}$, the curvature coefficient $(r=0.438 \pm 0.064)$ although large variations between segments and populations were observed (Table 5). We thus may conclude that lines clearly differ in their reactivity to temperature, some being more reactive than others.

For the sigmoid reaction norms of segments 6 and 7 , we calculated 7 characteristic values (Table 6). Among 28 correlation coefficients, only 11 were significantly positive. Three trait values ( $\operatorname{maxP}, \operatorname{minP}$ and Pip) were calculated and the most repeatable was maxP. Altogether the genetic repeatability was low $(0.332 \pm 0.109)$. The repeatability was better for the four plasticity values, on average $(0.473 \pm 0.088)$, especially for the Tip and the slope at the inflection point (0.49 and 0.59). This observation is in opposition with what has been observed for quadratic norms.

Genetic repeatability, as demonstrated here by a positive correlation between successive generations, is a convenient demonstration that phenotypic differences among lines do have a genetic component. However, the correlation between generations does not estimate the amount of genetic variablity harboured in the origin population (Gibert et al, 1998b). It was previously shown (Gibert et al, 1998b) that repeatability between family means was increased by increasing the number of flies measured in each line. From this kind of observation, it might be expected that the characteristic values of the reaction norms, which are estimated from the data of 70 flies, should be more precise, and thus more repeatable, than the family means, which are based on 10 flies only. Our results clearly showed such was not the case: repeatability of reaction norms characteristic values was, on average, slightly less than that of the family mean. It has been shown (Scheiner, 2002) that, for thorax and wing length, plasticity was genetically variable and could be selected for, although its heritability was much less than that of the traits. Moreover, we know that plasticity may be a target of natural selection and changes when tropical and temperate populations are compared (Delpuech et al, 1995; Morin et al, 1999; Moreteau et al, 2002). The fairly low repeatability of characteristic values of pigmentation reaction norms might be a consequence of an overall low heritability. The occurrence of some genetic variability in the shape of the curves suggests however that significant changes could be produced by directional selection. Also, the genetic variability of the characteristic values might now be investigated by a QTL (quantitative trait loci) procedure.

\section{References}

Agrawal AA (2001). Phenotypic plasticity in the interactions and evolution of species. Science 294: 321-326.

Callahan HS, Pigliucci M, Schlichting CD (1997). Developmental phenotypic plasticity: where ecology and evolution meet molecular biology. Bioessay 19: 519-525.

Capy P, Pla E, David JR (1994). Phenotypic and genetic variability of morphometrical traits in natural populations of Drosophila melanogaster and D. simulans. II. Withinpopulation variability. Genet Sel Evol 26: 15-28.

David JR, Capy P (1988). Genetic variation of Drosophila melanogaster natural populations. Trends Genet 4: 106-111.

David JR, Capy P, Gauthier JP (1990). Abdominal pigmentation and growth temperatures in Drosophila melanogaster: similarities and differences in the norms of reaction of successive segments. J Evol Biol 3: 429-445.

David JR, Capy P, Payant V, Tsakas S (1985). Thoracic trident pigmentation in Drosophila melanogaster: differentiation of geographical populations. Genet Sel Evol 17: 211-223.

David JR, Gibert P, Gravot E, Pétavy G, Morin JP, Karan D et al (1997). Phenotypic plasticity and developmental temperature in Drosophila: analysis and significance of reaction norms of morphometrical traits. J Therm Biol 22: 441-451.

David JR, Gibert P, Moreteau B (2004). Evolution of reaction norms. In: DeWitt TJ, Scheiner SM (eds) Phenotypic Plasticity. Functional and Conceptual Approaches. Oxford University Press: USA. pp 50-63.

David JR, Moreteau B, Gauthier JP, Pétavy G, Stockel J, Imasheva AG (1994). Reaction norms of size characters in relation to growth temperature in Drosophila melanogaster: an isofemale lines analysis. Genet Sel Evol 26: 229-251.

De Jong G (1995). Phenotypic plasticity as a product of selection in a variable environment. Am Nat 145: 493-512.

De Jong G, Gavrilets S (2000). Maintenance of genetic variation in phenotypic plasticity: the role of environmental variation. Genet Res Camb 76: 295-304.

Delpuech JM, Moreteau B, Chiche J, Pla E, Vouidibio J, David JR (1995). Phenotypic plasticity and reaction norms in temperate and tropical populations of Drosophila melanogaster: ovarian size and developmental temperature. Evolution 49: 670-675.

De Witt TJ, Scheiner SM (2004). Phenotypic Plasticity. Functional and Conceptual Approaches. Oxford University Press: USA.

Falconer DS (1989). Introduction to Quantitative Genetics. Longman: New York. 
Gibert P, Moreteau B, David JR (2000). Developmental constraints on an adpative plasticity: reaction norms of pigmentation in adult segments of Drosophila melanogaster. Evol Dev 2: 249-260.

Gibert P, Moreteau B, David JR, Scheiner SM (1998a). Describing the evolution of reaction norm shape: body pigmentation in Drosophila. Evolution 52: 1501-1506.

Gibert P, Moreteau B, Moreteau JC, David JR (1996). Growth temperature and adult pigmentation in two Drosophila sibling species: an adaptive convergence of reaction norms in sympatric populations? Evolution 50: 2346-2353.

Gibert P, Moreteau B, Moreteau JC, David JR (1998b). Genetic variability of quantitative traits in Drosophila melanogaster (fruit fly) natural populations: analysis of wild-living flies and of several laboratory generations. Heredity 80: 326-335.

Gibert P, Moreteau B, Moreteau JC, Parkash R, David JR (1998c). Light body pigmentation in Indian Drosophila melanogaster: a likely adaptation to a hot and arid climate. J Genet 77: 13-20.

Gotthard K, Nylin S (1995). Adaptive plasticity and plasticity as an adaptation: a selective review of plasticity in animal morphology and life history. Oikos 74: 3-17.

Hoffmann AA, Parsons PA (1988). The analysis of quantitative variation in natural populations with isofemale strains. Genet Sel Evol 20: 87-98.

Imasheva A, Moreteau B, David J (2000). Growth temperature and genetic variability of wing dimensions in Drosophila: opposite trends in two sibling species. Genet Res Camb 76: 237-247.

Karan D, Morin JP, Gravot E, Moreteau B, David JR (1999). Body size reaction norms in Drosophila melanogaster: temporal stability and genetic architecture in a natural population. Genet Sel Evol 31: 491-508.

Krebs R, Loeschke V (1995). Resistance to thermal stress in preadult Drosophila buzzatii: acclimation and variation among populations. Biol J Linn Soc 56: 505-515.

Moreteau B, Gibert P, Delpuech J-M, Pétavy G, David JR (2002). Phenotypic plasticity of sternopleural bristle number in temperate and tropical populaions of Drosophila melanogaster. Genet Res Camb 81: 25-32.

Morin JP, Moreteau B, Pétavy G, Imasheva S, David JR (1996). Body size and developmental temperature in Drosophila simulans: comparison of reaction norms with sympatric Drosophila melanogaster. Genet Sel Evol 28: 415-436.

Morin JP, Moreteau B, Pétavy G, David JR (1999). Divergence of reaction norms of size characters between tropical and temperate populations of Drosophila melanogaster and D. simulans. I Evol Biol 12: 329-339.

Munjal AK, Karan D, Gibert P, Moreteau B, Parkash R, David JR (1997). Thoracic trident pigmentation in Drosophila melanogaster: latitudinal and altitudinal clines in Indian populations. Genet Sel Evol 29: 601-610.

Pigliucci M (1998). Developmental phenotypic plasticity: where internal programming meets the external environment. Curr Opin Plant Biol 1: 87-91.

Pigliucci M (2001). Phenotypic Plasticity. Beyond Nature and Nurture. The Johns Hopkins University Press: Baltimore and London.

SAS (1985). SAS User's Guide: Statistics. SAS Institute, Inc.: Cary, NC.

Scheiner SM (1993a). Genetics and evolution of phenotypic plasticity. Ann Rev Ecol Systems 24: 35-68.

Scheiner SM (1993b). Plasticity as a selectable trait: reply to Via. Am Nat 142: 371-373.

Scheiner SM (2002). Selection experiments and the study of phenotypic plasticity. J Evol Biol 15: 889-898.

Scheiner SM, Lyman RF (1989). The genetics of phenotypic plasticity. I. Heritability. J Evol Biol 2: 95-107.

Schlichting CD, Pigliucci M (1993). Control of phenotypic plasticity via regulatory genes. Am Nat 142: 366-370.

Schlichting CD, Pigliucci M (1998). Phenotypic Evolution, a reaction norm perspective. Sinauer Associates, Inc.: Sunderland, MA.

Statistica (1999) Statistica StatSoft, Inc., Version 5.5, Tulsa.

van Dooren TJM (2001). Reaction norms with bifurcations shape by evolution. Proc Roy Soc London B 268: 279-287.

Van Tienderen PH, Koelewijn HP (1994). Selection on reaction norms, genetic correlations and constraints. Genet Res Camb 64: 115-125.

Via S (1992). Models of the evolution of phenotypic plasticity. Trends Ecol Evol 7: 63.

Via S (1993). Adaptive phenotypic plasticity: target or byproduct of selection in a variable environment? Am Nat 142: 352-365.

Via S (1994). The evolution of phenotypic plasticity: What do we really know?. Princeton University Press: Princeton, NJ.

Via S, Gomulkiewicz R, De Jong G, Scheiner SM, Schlichting CD, Van Tienderen PH (1995). Adaptive phenotypic plasticity: consensus and controversy. Trends Ecol Evol 5: 212-217. 